\title{
Automated Control Configuration Selection Considering System Uncertainties ${ }^{\dagger}$
}

\author{
Ali M. H. Kadhim, ${ }^{*,+}, \uparrow$ Miguel Castaño Arranz, ${ }^{\ddagger}$ and Wolfgang Birk ${ }^{\ddagger}$ \\ $\ddagger$ Control Engineering Group, Department of Computer Science, Electrical and Space \\ Engineering, Luleå University of Technology, Luleå, Sweden \\ ฯDepartment of Electrical Engineering, University of Kufa, Najaf, Iraq \\ E-mail: ali.kadhim@ltu.se
}

\begin{abstract}
This paper proposes an automated pairing approach for configuration selection of decentralized controllers which considers system uncertainties. Following the Relative Interaction Array (RIA) pairing rules, the optimal control configuration, i.e. the configuration that fits best the pairing rules, is obtained automatically by formulating the control configuration selection problem as an Assignment Problem (AP). In this AP, the associated costs related to each input-output pairing are given by the RIA coefficients. The Push-Pull algorithm is used to solve the AP for the nominal system and to obtain the set of costs for which the resulting configuration remains optimal, also called the perturbation set. The introduction of uncertainty bounds on the RIA-based costs enables the testing of the possible violation of the optimality conditions. Examples to illustrate the proposed approach for a $3 \times 3$ system and a $4 \times 4$ gasifier plant are given.
\end{abstract}

Keywords: Uncertain and multivariable systems, RIA uncertainty bounds, assignment problem, automatic optimal control configuration selection.

\footnotetext{
$\dagger$ 'Author's Post-print
} 


\section{Introduction}

Robustness against loop failures and simple design/upgrade are among features which favor the widely spread use of decentralized control strategies in chemical processes such as distillation column, ${ }^{1}$ pulp mill process,${ }^{2}$ waste water treatment plant, ${ }^{3}$ etc. An essential step in the design of decentralized control systems is finding the pairings of controlled and manipulated variables which lead to minimal loop interaction. This step is referred to as "input-output pairing" which is a possible approach for resolving the Control Configuration Selection (CCS) problem.

It is well known that large scale systems are hierarchically structured into optimization and control layers. ${ }^{4}$ The control layer is further decomposed into supervisory control (slow layer) where advance control like for example model predictive control is frequently used, and regulatory control (fast layer) where classical controllers like for example PID are employed. ${ }^{5}$ Selecting the best control configuration in a manual fashion among many available controlled and manipulated variables, in the supervisory and regulatory control layer, is a tedious and error-prone task. Moreover, neglecting the inherent uncertainty of system models in the configuration selection and base the selection solely on a nominal model might be inadequate. Uncertainties of the system model come from different sources such as parameter variation of the models due to nonlinearities or variations in the operating conditions, or neglecting system dynamics for the simplification of models, among others. ${ }^{4}$ The work in this paper provides an approach to automate the input-output pairing while taking the effect of system model uncertainties into account.

An approach to select control configurations is the use of Interaction Measures (IM) and their associated pairing rules. The first IMs date back to 1960's when Rijnsdorp introduced the Interaction Quotient ${ }^{6}$ and Bristol introduced the Relative Gain Array (RGA). ${ }^{7}$ Since then, many adaptations of the RGA as well as other IMs have been introduced to resolve the CCS problem for a larger class of systems. ${ }^{8}$ Moreover, uncertainty bounds for the RGA in the presence of model uncertainty where introduced by Chen and Seborg. ${ }^{9}$ Later, tighter 
bounds ${ }^{10}$ as well as more simplistic alternative bounds were proposed. ${ }^{11},{ }^{12}$ Additionally, bounds on the Dynamic RGA for $2 \times 2$ systems have been introduced. ${ }^{13}$

It is shown, to avoid performance degradation ${ }^{14}$ or even instability ${ }^{15}$ of the systems under a decentralized controller, the configuration has to be selected in a manner that minimizes the loop interaction. Using the RGA pairing rules there is no guarantee that the selected configuration yields a minimum level of overall interaction since the RGA quantifies the interaction for each control loop individually. ${ }^{16}$ Zhu addressed the problem of overall interaction reduction by introducing a new steady-state measure tool called Relative Interaction Array (RIA) along with new pairing criteria, ${ }^{17}$ which actually relates to the above mention interaction quotient. The new pairing rules also offer necessary conditions to satisfy closedloop stability, integrity and robustness. Therefore, a decision has been made to base the optimal configuration selection on the pairing that fits the RIA pairing rules best.

The RGA-number ${ }^{4}$ can be seen as an automatic configuration selection tool based on the RGA matrix. However, the RGA-number needs to be re-calculated for each possible configuration, which might lead to large computational efforts as the size of the system increases. To reduce the computational efforts of finding the optimal pairings for large-scale systems, Kariwala and Cao ${ }^{18}{ }^{19}$ proposed the branch and bound (BAB) approach in which the RGA and the $\mu$-interaction measure ( $\mu$-IM) are used as selection criteria. There, lower bounds of the RGA-number and the $\mu$-IM are derived and used in pruning the branches of the solution tree that do not lead to optimal solution. A systematic algorithm to obtain candidate pairings based on RGA and RIA properties was proposed by Kookos and Lygeros. ${ }^{20}$ The basic idea behind that algorithm is formulating the problem of finding the configuration that minimizes the interaction as a Mixed Integer Linear Programming (MILP) problem. In a similar manner, Fatehi formulated the configuration selection problem as an Assignment Problem (AP) ${ }^{21}$ leading to a method for automatic pairing selection. In this AP, the costs associated to the assignment of the pairings is given by a pairing measure called Normalised RGA (NRGA). The NRGA is obtained by means of a nonlinear map which maps the RGA 
values on the $[0,1]$ subspace. ${ }^{21}$ Yet the literature lacks bridging the automated configuration selection with the effect of system uncertainties.

In addition to the methods based on relative gains, there are other families of CCS tools. These include i) the gramian-based IMs, ii) methods using optimization techniques, ${ }^{22}$ iii) methods for plat-wide control, ${ }^{23}$ or iv) methods for the reconfiguration of control systems. ${ }^{24}$ Perhaps, the most relevant family to survey in relation to the IMs based on relatives gains are the gramian-bsed IM. The gramian-based IMs can be used to design sparse configurations and use models with frequency-domain information. The gramin-based IMs have received increasing attention since the introduction of the Participation Matrix (PM) by M. Salgado ${ }^{25}$ and the posterior introduction of HIIA ${ }^{26}$ and $\Sigma_{2} \cdot{ }^{27} \mathrm{~A}$ compelling aspect of these IMs, is that gramians are the subject of a large volume of literature, and advances on gramians are likely to have a straightforward application on CCS, like the use of the cross-gramian matrix for bilinear systems. ${ }^{28}$ The gramian-bsed IMs compress frequency domain data in a single measure, and therefore it is traditional to restrict the range of frequencies of interest with e.g. the use of pref-filters. ${ }^{27} \mathrm{~A}$ traditional alternative to the filters is the straightforward use of the frequency limited gramians defined in 1990 and which form part of the MATLAB since 2006. The use of either filters ${ }^{27,29}$ or the frequency-limited gramians ${ }^{30}$ are equivalent alternatives with the use of perfect filters. ${ }^{31}$ Additionally, there are a number of studies on bounds of gramian-based IMs from uncertain models or directly from process data, including bounds on PM, ${ }^{32-36}$ HIIA $^{32}$ and $\Sigma_{2} \cdot{ }^{35-37}$ These bounds can be considered in the decision making using an algorithm for automatic CCS. ${ }^{36}$

In this article an approach for automated control configuration selection considering the system uncertainties is proposed. It excludes the configurations that lead to loss of stability and integrity before looking for the optimal configuration among the rest. Basically, the optimal control configuration is achieved from the nominal system at steady-state using AP algorithm, namely the Push-Pull algorithm following the RIA pairing rules. The Push-Pull algorithm also provides information to determine the perturbation set within which the opti- 
mality of the configuration is preserved. A verification step is to be performed to demonstrate whether the optimal configuration is still the optimum one for the uncertain system or not. Due to RIA stability and integrity requirements, the Push-Pull algorithm might indicate that there is no feasible decentralized configuration which can stabilize the process with integrity. This method will extend the capabilities of the software tool ProMoVis ${ }^{38}$ which currently depends on the manual pairing selection by the user.

The structure of the paper is as follows. First, conceptual overview of the proposed approach is given in Section 2 followed by a brief description for the RIA and its pairing rules in Section 3. In Section 4, the relationship between the CCS and the AP, the perturbation set and the automated configuration selection are stated. The uncertainty bounds of the RIA are derived in Section 5. In Section 6 the change in the absolute RIA is formulated as a polytopic model and the proposed approach is presented as an algorithm in Section 7. Illustration of the proposed approach using examples is given in Section 8. The article is closed with conclusions and suggested future work in Section 9.

\section{Conceptual Overview}

In the proposed approach, the optimal configuration selection is achieved automatically using the Push-Pull algorithm according to the RIA pairing rule of minimizing the overall interaction. However, the RIA elements with lower uncertainty bounds not satisfying the requirements for the stability and integrity are removed before performing the Push-Pull algorithm. Thus, the optimal control configuration in this article is understood as the configuration that minimizes the overall interaction as well as fulfils the stability and integrity requirements based on the RIA definition. The pairing based on the RIA criteria is adopted here since it does not only offer a comprehensive solution for the configuration selection but also fits the AP terminology without modification. Then, utilizing a perturbation set, the proposed approach verifies whether the optimal configuration selection is still optimum 
under particular system uncertainties or if a change is expected. The perturbation set is determined using information obtained as a by-product of the Push-Pull algorithm.

The following phases summarize the core of the proposed approach:

(i) Obtaining the optimal configuration selection automatically based on the nominal system at steady-state and the uncertainty bounds of the RIA elements using the PushPull algorithm. However, by removing the pairs that might lead to lose stability and integrity, there is a chance the Push-Pull algorithm gives no solution. Such a case means that there is no feasible configuration and thus no decentralized controller can satisfy the stability and integrity requirements for the uncertain system (see Section 4 and Section 5).

(ii) Obtaining the perturbation set for which the optimal configuration based on the nominal system is still the optimum for uncertain system. The Push-Pull algorithm provides information to produce the halfspace equations from which the perturbation set is determined (see subsection 4.2).

(iii) Calculating the uncertainty bounds of the absolute RIA caused by the uncertainty in the system gains. The information provided by RIA uncertainty bounds is to be used in the verification phase (see the details in Section 5).

(iv) Verifying whether the calculated uncertainty bounds of the absolute RIA obtained in (iii) satisfy the perturbation set determined in $(i i)$. If so, the optimal configuration selection obtained in $(i)$ is optimum for the perturbed system; otherwise it still satisfies the stability and integrity conditions for the perturbed system but does not minimize the overall interaction (see Section 6 for the details).

It is worth mentioning that the Push-Pull algorithm in $(i)$ is performed only once on the RIA matrix obtained from the nominal system in order to find the optimal pairing, if there is any. In addition, the difference between the absolute nominal and perturbed RIA values is written in a polytopic form to simplify the verification process in $(i v)$. 
Using simple mathematical operations, the vertices of the polytope are checked for satisfying the perturbation set and a conclusion can be drawn regarding the preservation of the pairing optimality.

\section{RIA and its Pairing Rules}

Consider a multivariable transfer function $G(s)$ with input vector $u \in \mathcal{C}^{n}$ and output vector

$y \in \mathcal{C}^{n}$ under decentralized control. For a Single-Input-Single-Output (SISO) control loop connecting the pair $\left(y_{i}-u_{j}\right)$, a change in $u_{j}$ would influence $y_{i}$ directly through the elementary transfer function $\left(g_{i j}\right)$ and indirectly through the possible loop interaction. ${ }^{16}$ Therefore, the response of $y_{i}$ to the change can be expressed as

$$
y_{i}(s)=\left[g_{i j}(s)+a_{i j}(s)\right] u_{j}(s)
$$

where $a_{i j}$, the absolute interaction, represents the effect of the other control loops on the $\left(y_{i}-u_{j}\right)$ pair due to loop interaction. ${ }^{16}$

Defining the relative interaction ${ }^{17}$ as

$$
\phi_{i j}(s)=\frac{a_{i j}(s)}{g_{i j}(s)}
$$

the response of $y_{i}$ in $(1)$ is written ${ }^{8}$ as

$$
y_{i}(s)=g_{i j}(s)\left[1+\phi_{i j}(s)\right] u_{j}(s)=\tilde{g}_{i j}(s) u_{j}(s)
$$

where the term $\left[1+\phi_{i j}(s)\right]$ measures the amplification or attenuation in the $g_{i j}$ due to loop interaction effect. Following the determination of $a_{i j}$ using the signal-flow graph technique, $\phi_{i j}$ can be obtained. ${ }^{16}$ The steady-state $a_{i j}(0)^{17}$ is defined as (seeking simplicity, the (0) of 
the steady-state is dropped and will only be used where needed)

$$
a_{i j}=\frac{1}{\left[G^{-1}\right]_{j i}}-g_{i j}
$$

under the following assumptions

1. the closed-loop system has integrity against any single-loop failure, and

2. each controller contains an integral action in each control loops.

Hence, from (2) and (4), the RIA matrix $(\Phi)$ is calculated in terms of its $i j$-elements as

$$
[\Phi]_{i j}=\frac{1}{g_{i j}\left[G^{-1}\right]_{j i}}-1=\frac{1}{[\Lambda]_{i j}}-1
$$

where $\Lambda$ is the RGA matrix calculated by

$$
\Lambda=G \times\left(G^{-1}\right)^{T}
$$

with $\times$ and $T$ denote element-by-element multiplication and matrix transpose, respectively.

Finally, RIA pairing rules state that ${ }^{17}$ the input-output variables should be paired so that

(a) Niederlinski Index (NI) $>0$ (stability rule).

(b) $\phi_{i j}>-1$ (integrity rule).

(c) avoiding $\phi_{i j}$ close to -1 (robustness rule), and

(d) $\min \sum\left|\phi_{i j}^{k}\right|$ (overall interaction rule).

where $\phi_{i j}^{k}$ represents the pairing elements corresponding to the $k^{t h}$ possible pairing that satisfies the first three rules. Notice that, the stability condition $(N I>0)$ is fulfilled by avoiding pairs corresponding to $\phi_{i j} \leq-1 .{ }^{17}$ Thus, in order to satisfy stability and integrity conditions, pairs corresponding to $\phi_{i j} \leq-1$ have to be avoided. 
Direct extension of (4), (5) and the pairing rules to frequencies other than the steadystate seems a straightforward approach, but has not been proven to be valid for the RIA. One of the basic assumptions for the RIA and also the RGA is the perfect control condition, which in turn might lead to inaccuracies. ${ }^{16}, 17$ Thus, the proposed approach is only applied in steady-state case.

\section{Assignment Problem, Push-Pull algorithm and Auto- mated Control Configuration Selection}

In this section a description of the AP is introduced and the reason for choosing the PushPull algorithm to solve the AP is explained. Later, the formulation of the CCS problem as an $\mathrm{AP}$ is given.

\subsection{The Assignment Problems}

The Assignment Problem (AP) is a special type of the Transportation Problem (TP). The TP deals with finding the minimum cost of transporting products from several manufactures to several consumers whereas the output of the AP is assigning one manufacturer to one and only one consumer so that the transportation cost is minimized. With $\left(c_{i j}\right)$ being the cost of conveying a single product from manufacturer $(i=1, . ., n)$ to consumer $(j=1, . ., n)$, the $\mathrm{AP}$ is represented mathematically ${ }^{39}$ as

$$
\begin{gathered}
\min \sum_{i=1}^{n} \sum_{j=1}^{n} c_{i j} x_{i j} \\
\text { subject to } \sum_{j=1}^{n} x_{i j}=1 \text { for } i=1, \cdots, n \\
\text { and } \sum_{i=1}^{n} x_{i j}=1 \text { for } j=1, \cdots, n \\
\text { where } x_{i j}=0 \text { or } 1 \text { for all } i \text { and } j
\end{gathered}
$$


In the above, the one and only one condition is represented in the first and second constraints.

The control configuration selection is analogous to the AP, in the sense that the designer aims at selecting the input-output pairs (assigning one input to one and only one output) that minimizes the effect of the loop interaction, equivalent to the cost in the TP terminology.

\subsection{Push-Pull Algorithm and Perturbation Set}

Although the AP is a special type of Linear Programming (LP) optimization problem which is usually solved by utilizing the Simplex algorithm, ${ }^{39}$ methods such as Stepping-Stone and Hungarian algorithm are widely used to get the optimal solution. ${ }^{40}$ The sensitivity analysis is used to investigate the effect of changing $c_{i j}$ in (7) on that optimal solution. However, Stepping-Stone and Hungarian algorithms do not provide useful information to perform the sensitivity analysis for the AP due to an inherent degenerated optimal solution (degenerated when right-hand-side $(R H S)=0) .{ }^{40}$ In that direction, Adlakha and Arsham proposed the Push-Pull algorithm which provides a solution to the AP and enough information to determine the perturbation set for which the optimal solution is preserved. ${ }^{40}$

For a thorough understanding of the Push-Pull algorithm, the reader is referred to the work of Adlakha et al. ${ }^{40}$ and Arsham et al. ${ }^{41,42}$ In general, the Push-Pull algorithm is a Simplex type algorithm that consists of an Initialization phase as well as Push and Pull iterative phases. In the Initialization phase an initial tableau with some basic variables (BV) is generated. The BV are members of the basic variable set (BVS), which represents the solution of the AP given in (7). Throughout the Push phase, the BVS in the initial tableau is being contentiously filled with basic variables while being pushed towards the optimum corner. However, the feasibility may not be preserved in the Push phase $(R H S<0)$, in such a case, the Pull phase pulls back the BVS to the feasible corner. ${ }^{40}$ Eventually, the Push-Pull algorithm provides the optimal solution of (7) (i.e. BVS) including the information necessary for calculating the perturbation set $(\theta)$ from the final tableau. The perturbation set $(\theta)$ is 
determined as a function of the change in the cost $c_{i j}\left(\dot{c}_{i j}\right)$ in the AP given in (7) as

$$
\theta=\left\{\dot{c}_{i j} \mid C_{N}-C_{B} \cdot[A] \geq 0\right\}
$$

where $C_{B}$ is a row vector comprising the new costs corresponding to the basic variables set (BVS) while $C_{N}$ is a row vector comprising the new costs corresponding to the non-basic variables (non-BV). $[\mathrm{A}]$, on the other hand, denotes a matrix/vector obtained from the final tableau of the Push-Pull algorithm. To preserve the optimal solution of (7) for the perturbed case, the change in the costs has to satisfy the perturbation set $(\theta)$.

\subsection{RIA-Based Automated Configuration}

Since $x_{i j}$ are either zeros or ones for the AP as in (7), $\left|\phi_{i j}^{k}\right|$ in RIA pairing rule (d) given in Section 3 , is analogue to the $c_{i j}$ in (7). Thus, pairing rule (d) can be rewritten as

$$
\begin{aligned}
& \min _{\phi_{i j} \in \Omega} \sum_{i=1}^{n} \sum_{j=1}^{n}\left|\phi_{i j}\right| x_{i j} \\
& \text { subject to } \sum_{j=1}^{n} x_{i j}=1 \text { for } i=1, \cdots, n \\
& \text { and } \sum_{i=1}^{n} x_{i j}=1 \text { for } j=1, \cdots, n \\
& \text { where } x_{i j}=0 \text { or } 1 \text { for all } i \text { and } j
\end{aligned}
$$

where $\Omega=\left\{\phi_{i j}: \phi_{i j}>-1\right\}$.

Notice that, the $n^{\prime} \mathrm{s}$ in (9) represent the system size rather than the number of the manufacturers and consumers in (7).

The Push-Pull algorithm is hence used to assign values of 1 to the $x_{i j}$ in (9) that correspond to the optimal $\left|\phi_{i j}\right|$ that would lead to the minimization of the overall loop interaction.

The optimal $\left|\phi_{i j}\right|$ are the $\phi^{\prime} s$ with values close to zero in order to keep the term $\left[1+\phi_{i j}\right]$ in (3) as close as possible to 1 . In other words, the pairs that corresponds to $x_{i j}=1$ are 
selected such that the loop interaction effect is minimized by keeping $\tilde{g}_{i j}$ in (3) as close as possible to $g_{i j}$. Moreover, the closed-loop stability and integrity conditions in the respective rules (a) and (b) is to be fulfilled by removing the pairs corresponding to the $\phi_{i j} \leq-1$ from the initial tableau before performing the Push-Pull algorithm. Thus, the automated optimal configuration selection satisfies the stability condition by default in contrast to the approach presented by Fatehi ${ }^{21}$ which proposes switching to the suboptimal pairing if the optimal one does not satisfy the stability condition. As for rule (c), it is satisfied since the pairs corresponding to the $\phi_{i j}$ close to zero will be selected while performing the Push-Pull algorithm. However, removing the pairs corresponding to the $\phi_{i j} \leq-1$ from the initial tableau is valid for the unperturbed systems. For perturbed systems $G_{P}$, removing the pairs correspond to the perturbed RIA $\left(\phi_{P i j}\right)$ with lower bounds $\leq-1$ has to be considered. The uncertainty bounds of the RIA elements are derived in the next section.

\section{RIA Uncertainty Bounds}

Many sources lead to model uncertainties such as process perturbations, linear approximations around specific operating points, or neglecting higher order dynamics. Chen and Seborg ${ }^{9}$ had revealed the importance of considereing model uncertainties in the pairing selection. Prior to the application of the introduced automatic pairing method in Section 4, it is required to derive the uncertainty bounds on the RIA caused by the uncertainties of the system gains. These bounds are used to: i) satisfy the closed-loop stability and integrity requirements for uncertain systems following pairing rules (a) and (b), by removing from the feasibility set those pairings that correspond to RIA elements having lower bound $\leq-1$. ii) check if the optimality of the configuration obtained with the automatic pairing is preserved for perturbed system.

Using Taylor's series expansion and by truncating after the first-order term, the perturbed 
$i j-$ element in RIA matrix $\left(\phi_{P i j}\right)$ can be approximated as

$$
\phi_{P i j} \approx \phi_{i j}+\sum_{k=1}^{n} \sum_{l=1}^{n}\left(\frac{\partial \phi_{i j}}{\partial g_{k l}}\right)_{G}\left(\delta g_{k l}\right)
$$

where $\phi_{i j}$ is the $i j$-element in RIA calculated based on the nominal system $G, \delta g_{k l}$ is the difference between the perturbed and nominal $k l$-element of $G$ and $\frac{\partial \phi_{i j}}{\partial g_{k l}}$ is given as in (11) (see the full derivation in the Appendix)

$$
\frac{\partial \phi_{i j}}{\partial g_{k l}}= \begin{cases}-\frac{\phi_{i j}}{g_{i j}} & i=k \text { and } j=l \\ \frac{\left(\phi_{i j}+1\right)}{\left(\phi_{k l}+1\right) g_{k l}} & i=k \text { or } j=l \\ \left(-\frac{(-1)^{i+j+k+l} g_{i j} \operatorname{det}\left(G^{i j, k l}\right)\left(\phi_{i j}+1\right)}{\operatorname{det}(G)}+\frac{1}{\left(\phi_{k l}+1\right) g_{k l}}\right)\left(\phi_{i j}+1\right) & i \neq k \text { and } j \neq l\end{cases}
$$

Taking the absolute value of both sides, (10) is re-written as

$$
\left|\phi_{P i j}-\phi_{i j}\right|=\left|\sum_{k=1}^{n} \sum_{l=1}^{n}\left(\frac{\partial \phi_{i j}}{\partial g_{k l}}\right)_{G}\left(\delta g_{k l}\right)\right|
$$

Using the fact $|a+b| \leq|a|+|b|$, (12) can be written as

$$
\left|\phi_{P i j}-\phi_{i j}\right| \leq \sum_{k=1}^{n} \sum_{l=1}^{n}\left|\left(\frac{\partial \phi_{i j}}{\partial g_{k l}}\right)_{G}\left(\delta g_{k l}\right)\right|
$$

The uncertainty bounds of the perturbed RIA elements $\left(\phi_{P i j}\right)$ can be found by applying $|a| \leq b \Leftrightarrow-b \leq a \leq b$ on (13) as

$$
\overbrace{\phi_{i j}-\sum_{k=1}^{n} \sum_{l=1}^{n} \mid\left(\frac{\partial \phi_{i j}}{\partial g_{k l}}\right)_{G}\left(\delta g_{k l}\right)}^{\phi_{P i j}} \mid \leq \phi_{P i j} \leq \underbrace{\phi_{i j}+\sum_{k=1}^{n} \sum_{l=1}^{n}\left|\left(\frac{\partial \phi_{i j}}{\partial g_{k l}}\right)_{G}\left(\delta g_{k l}\right)\right|}_{\overline{\phi_{P i j}}}
$$


Accordingly, (9) has been adjusted for the perturbed systems as

$$
\begin{aligned}
\min _{\phi_{i j} \in \Omega_{P}} & \sum_{i=1}^{n} \sum_{j=1}^{n}\left|\phi_{i j}\right| x_{i j} \\
\text { subject to } & \sum_{j=1}^{n} x_{i j}=1 \text { for } i=1, \cdots, n \\
\text { and } & \sum_{i=1}^{n} x_{i j}=1 \text { for } j=1, \cdots, n
\end{aligned}
$$

where $x_{i j}=0$ or 1 for all $i$ and $j$

where $\Omega_{P}=\left\{\phi_{i j}: \underline{\phi_{P i j}}>-1\right\}$.

Since rule (d) involves the absolute RIA values, the bounds of $\left|\phi_{P i j}\right|$ have to be engaged in the optimality verification. The uncertainty bounds of $\left|\phi_{P i j}\right|$ are derived using the definition of the absolute function as in (18) after rewriting (14) in an affine parameter-dependent model as

$$
\phi_{P i j}=\phi_{i j}+\delta \phi_{i j}
$$

where $\delta \phi_{i j}$ is bounded as

$$
\begin{gathered}
\overbrace{-\sum_{k=1}^{n} \sum_{l=1}^{n}\left|\left(\frac{\partial \phi_{i j}}{\partial g_{k l}}\right)_{G}\left(\delta g_{k l}\right)\right|}^{\delta_{i j}} \leq \delta \phi_{i j} \leq \underbrace{n}_{\overline{\sum_{i j}} \sum_{l=1}^{n}\left|\left(\frac{\partial \phi_{i j}}{\partial g_{k l}}\right)_{G}\left(\delta g_{k l}\right)\right|} \\
\left\{\begin{array}{l}
0 \leq\left|\phi_{P i j}\right| \leq \max \left(\left|\phi_{i j}+\underline{\delta \phi_{i j}}\right|,\left|\phi_{i j}+\overline{\delta \phi_{i j}}\right|\right) \quad \text { if } \phi_{i j}+\overline{\delta \phi_{i j}} \geq 0 \text { and } \phi_{i j}+\underline{\delta \phi_{i j}} \leq 0 \\
\min \left(\left|\phi_{i j}+\underline{\delta \phi_{i j}}\right|,\left|\phi_{i j}+\overline{\delta \phi_{i j}}\right|\right) \leq\left|\phi_{P i j}\right| \leq \max \left(\mid \phi_{i j}+\underline{\left.\delta \phi_{i j}|,| \phi_{i j}+\overline{\delta \phi_{i j}} \mid\right) \quad \text { otherwise }}\right.
\end{array}\right.
\end{gathered}
$$

Notice that (14) will be used to satisfy the pairing rules (a) and (b) for the uncertain systems by avoiding the pairs correspond to $\phi_{P i j} \leq-1$ while the upcoming equations $(21)$ and 
(22) which are derived from (18) are the ones to be used to verify the validly of the optimal configuration obtained from the nominal system $(G)$ under the effect of the uncertainty.

The uncertainty bounds of $\phi_{P i j}$ given by (14), following the Chen and Seborg ${ }^{9}$ approach, are rather conservative. For curious readers, an alternative method to calculate the uncertainty bounds of RIA elements $\left(\phi_{i j}\right)$, rather than (14), is discussed in Appendix.

However, the proposed approach is designed such that it does not depend on specific uncertainty bounds for the RIA. In that sense better bounds could be easily integrated instead of the currently used ones.

\section{The Polytopic Model and the Model Vertices}

As seen from (8), the perturbation set $(\theta)$ is a function of the change in the cost $\left(c_{i j}\right)$. Notice that, the transportation individual costs $\left(c_{i j}\right.$ 's) in the AP given in (7) are usually either increased or decreased by a certain amount and it rarely happens that all of them are changed at the same time unless there is a market crises. Thus, verifying the preservation of the optimal solution of (7) is rather easy in the marketing field. On the contrary, a change in a single system gain $\left(g_{i j}\right)$ will affect all elements of the RIA matrix and produce an uncertainty bound around each of them as one can see from (14). As a result, an efficient way is needed to account for the effect of the change in the system gains on the absolute RIA elements and to simplify the verification of whether the change in the absolute RIA elements $\left(\left|\phi_{P i j}\right|-\left|\phi_{i j}\right|\right)$ satisfies the perturbation set $(\theta)$.

Therefore, the change in the absolute RIA matrix, $\left|\Phi_{P}\right|-|\Phi|$, is chosen to be written in an

affine parameter-dependent model which can be easily represented by a polytopic model. ${ }^{43}$ Since the polytopic model of the change in the absolute RIA matrix and the halfspaces of the perturbation set $(\theta)$ constitute two convex sets, it is convenient to draw a conclusion about the preservation of the pairing optimality by checking whether the vertices of the polytopic model satisfy the perturbation set. More specifically, if all possible vertices satisfy 
the perturbation set $(\theta)$, the optimal configuration obtained from the nominal system is still the optimal for the uncertain system. Otherwise, the optimality of the configuration for the uncertain system is not guaranteed.

The affine parameter-dependent model of the change of the absolute of RIA can be written after taking the absolute, generalizing and adding $(-|\Phi|)$ to both sides of (16) as

$$
\left|\Phi_{P}\right|-|\Phi|=\sum_{i=1}^{n} \sum_{j=1}^{n} P_{i j} S_{i j}
$$

and $P_{i j}$ is bounded as

$$
\underline{P_{i j}} \leq P_{i j} \leq \overline{P_{i j}}
$$

where $\underline{P_{i j}}$ and $\overline{P_{i j}}$ are given in (21) and (22), respectively.

$$
\begin{aligned}
& \underline{P_{i j}}= \begin{cases}-\left|\phi_{i j}\right| & \text { if } \phi_{i j}+\overline{\delta \phi_{i j}} \geq 0 \text { and } \phi_{i j}+\underline{\delta \phi_{i j}} \leq 0 \\
\min \left(\left|\phi_{i j}+\underline{\delta \phi_{i j} \mid},\right| \phi_{i j}+\overline{\delta \phi_{i j}} \mid\right)-\left|\phi_{i j}\right| & \text { otherwise }\end{cases} \\
& \overline{P_{i j}}=\max \left(\left|\phi_{i j}+\underline{\delta \phi_{i j}}\right|,\left|\phi_{i j}+\overline{\delta \phi_{i j}}\right|\right)-\left|\phi_{i j}\right| \quad \text { everywhere }
\end{aligned}
$$

In (19), $S_{i j}$ is an $n \times n$ matrix with $(i j)$-element equals to 1 and all other elements equal to 0 , for example $S_{11}$ and $S_{21}$ in a $3 \times 3$ system would look like

$$
S_{11}=\left[\begin{array}{lll}
1 & 0 & 0 \\
0 & 0 & 0 \\
0 & 0 & 0
\end{array}\right], S_{21}=\left[\begin{array}{lll}
0 & 0 & 0 \\
1 & 0 & 0 \\
0 & 0 & 0
\end{array}\right]
$$

The matrix $S_{i j}$ is imposed to make the sides of (19) consistent as the left side is written in a matrix form while the right side is expressed by the $P^{\prime} s$ of the $(i j)$-elements.

To aggregate all the possible vertices $\left(v_{1}, v_{2}, \cdots, v_{2^{n \times n}}\right)$ of the model (19) resulted from 
the variations of $P_{i j}$ for $i, j=1,2, \ldots, n$, a binary counter style is employed since each $P_{i j}$ is bounded by a low and a high value, i.e $\underline{P_{i j}}$ and $\overline{P_{i j}}$.

Thus, the vertices $\left(v_{1}, v_{2}, \cdots, v_{2^{n \times n}}\right)$ are written as

$$
v_{1}=\left[\begin{array}{c}
\frac{P_{11}}{\underline{P_{12}}} \\
\frac{P_{13}}{\vdots} \\
\underline{P_{n n}}
\end{array}\right], v_{2}=\left[\begin{array}{c}
\overline{P_{11}} \\
\frac{P_{12}}{P_{13}} \\
\vdots \\
\underline{P_{n n}}
\end{array}\right], \cdots, v_{2^{n \times n}}=\left[\begin{array}{c}
\overline{P_{11}} \\
\overline{P_{12}} \\
\overline{P_{13}} \\
\vdots \\
\overline{P_{n n}}
\end{array}\right]
$$

However, the number of vertices is reduced by the number of omitted $\phi_{i j}$ correspond to $\underline{\phi_{P i j}} \leq-1$. Thus, the user has to check $2^{(n \times n)-n_{\phi_{P i j}} \leq-1}$ vertices, where $n_{\phi_{P i j} \leq-1}$ denotes the number of the omitted $\phi_{i j}$.

\section{Algorithm of the Proposed Approach}

An algorithm that summarizes the approach and its prerequisites is given as follows and demonstrated by a flowchart as shown in Figure 1 .

\section{Examples}

The proposed approach is elucidated in this section by means of two examples. The first deals with the approach's Output1 and Output2 while in the second, a steady-state gain matrix of ALSTOM gasifier plant is used to discuss Output3. 


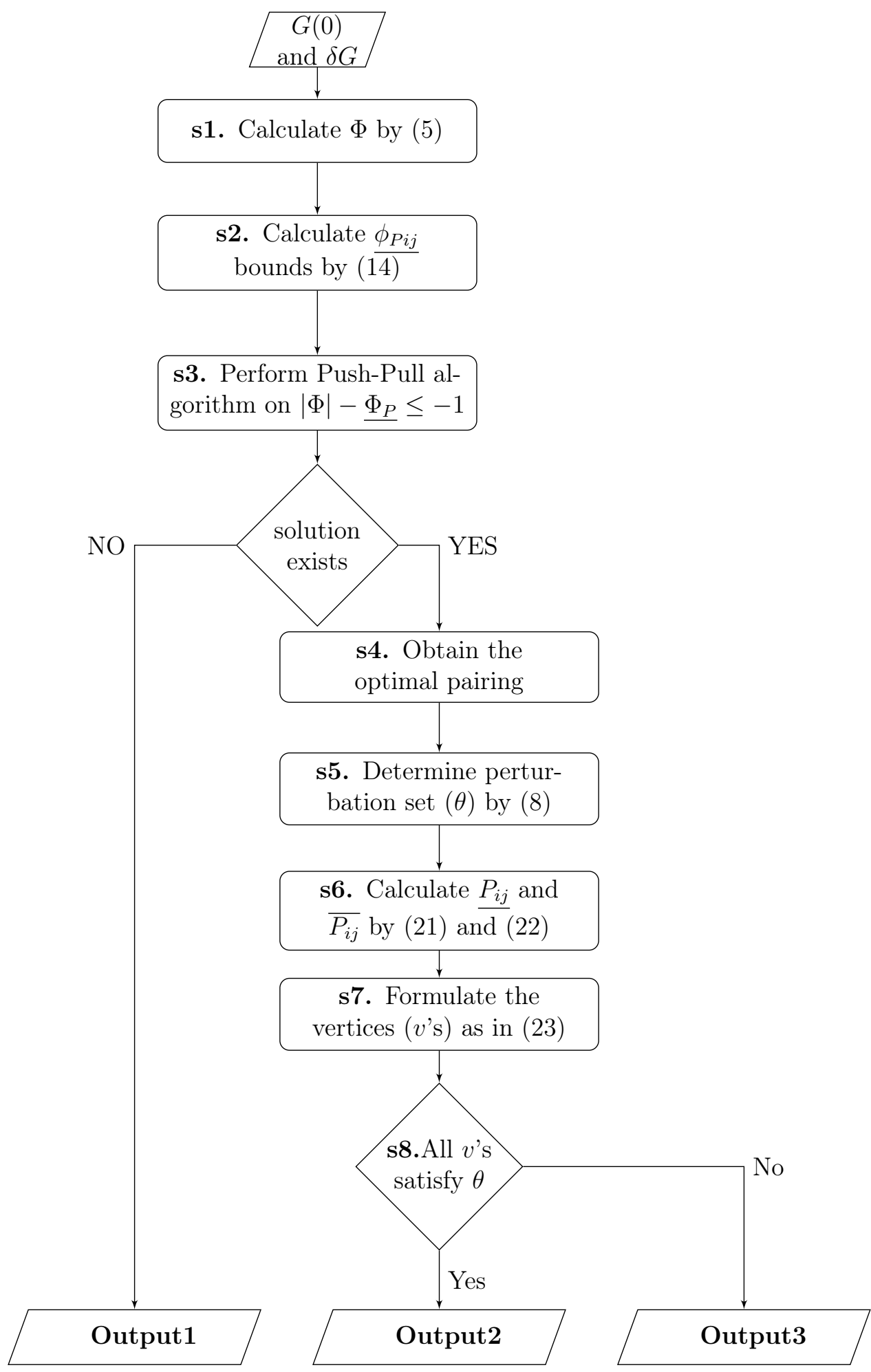

Figure 1: Flowchart of the proposed approach where the trapezoidal blocks refer to the input and outputs information and $\mathbf{s}$ is an abbreviation for step. Output1 refers to no decentralized controller stabilizes $G_{P}$ exists. Output 2 refers to the optimal configuration selection obtained in step 4 is still optimum for $G_{P}$. Output3 refers to the configuration obtained in step 4 satisfies the stability and integrity conditions for $G_{P}$ but minimizing the overall interaction is not guaranteed. 


\section{The algorithm of the proposed approach}

Input. The steady-state gain matrix of the nominal system $G(s)$ and the system uncertainty $\delta G$. step 1. Calculate RIA $(\Phi)$ using $G(0)$ by (5).

step 2. Calculate the lower uncertainty bound of the perturbed RIA elements $\left(\phi_{P i j}\right)$ by (14) for $i, j=1,2, \ldots, n$.

step 3. Perform the Push-Pull algorithm on $|\Phi|$ after omitting all $\phi_{i j}$ correspond to $\phi_{P i j} \leq-1$, if no solution exists

then Output1 no decentralized controller stabilizes the uncertain system $\left(G_{P}\right)$ exists. else do

step 4. Obtain the optimal configuration selection for the nominal system $G$.

step 5. Determine the perturbation set $(\theta)$ by $(8)$.

step 6. Calculate $P_{i j}$ and $\overline{P_{i j}}$ for $i, j=1,2, \ldots, n$ (except for those correspond to $\phi_{P i j} \leq-1$ ) through $(\overline{21)}$ and (22).

step 7. Formulate the vertices as in (23).

step 8. Verify whether the vertices in (23) satisfy the perturbation set $(\theta)$ determined from step $\mathbf{5}$, if all vertices satisfy the perturbation set $(\theta)$

then Output2 the optimal configuration selection obtained in step 4 is still optimum for the perturbed system $\left(G_{P}\right)$.

else Output3 the configuration obtained in step 4 satisfies the stability and integrity conditions for $G_{P}$ but minimizing the overall interaction is not guaranteed.

\subsection{Example 1}

Consider the following nominal system given by

$$
G(s)=\left[\begin{array}{ccc}
\frac{-2}{10 s+1} & \frac{1.5}{s+1} & \frac{1}{s+1} \\
\frac{1.5}{s+1} & \frac{1}{s+1} & \frac{-2}{10 s+1} \\
\frac{1}{s+1} & \frac{-2}{10 s+1} & \frac{1.5}{2 s+1}
\end{array}\right]
$$

and an additive uncertainty is given by

$$
|\delta G| \leq \alpha|G(0)|
$$

with $\alpha=0.01$.

Applying step 1, the $\Phi$ matrix is obtained as 


$$
\Phi=\left(\begin{array}{ccc}
-2.0750 & -0.1569 & 0.3437 \\
-0.1569 & 0.3438 & -2.0750 \\
0.3437 & -2.0750 & -0.1569
\end{array}\right)
$$

The lower uncertainty bound of the perturbed RIA elements are calculated as in step 2 using (14). The lower uncertainty bound values $\left(\phi_{P i j}\right)$ are aggregated in the following matrix

$$
\underline{\Phi_{P}}=\left(\begin{array}{ccc}
-2.2253 & -0.2287 & 0.2282 \\
-0.2287 & 0.2282 & -2.1924 \\
0.2282 & -2.1924 & -0.2118
\end{array}\right)
$$

As one can notice, $\underline{\phi_{P 11}}, \underline{\phi_{P 23}}$ and $\underline{\phi_{P 32}}$ have values $<-1$ and thus the configurations that involve any pair corresponds to $\phi_{11}, \phi_{23}$ or $\phi_{32}$ are to be removed in step 3 by eliminating the columns $x_{11}, x_{23}$ and $x_{32}$ from the initial tableau. In that way, by applying the Push-Pull algorithm on $|\Phi|$, the user is seeking the configuration which minimizes the loop interaction effect among only the configurations that satisfy the stability and integrity conditions. Not surprisingly, a solution to the Push-Pull algorithm in this case is expected to exist since 2 configurations are left after removing the ones which may lead to lose stability and integrity. The output of the Push phase is shown in Table 1. Since all the RHS elements are non Table 1: Final tableau

\begin{tabular}{ccc}
\hline BVS & $x_{22}$ & $R H S$ \\
\hline$x_{12}$ & 1 & 1 \\
$x_{21}$ & 1 & 1 \\
$x_{33}$ & 1 & 1 \\
$x_{13}$ & -1 & 0 \\
$x_{31}$ & -1 & 0 \\
\hline
\end{tabular}

negative, there is no need to perform the Pull phase. Thus, the optimum configuration 
selection corresponds to BV $x_{12}, x_{21}$ and $x_{33}$ in the final tableau, congruous to $y_{1}-u_{2}, y_{2}-u_{1}$ and $y_{3}-u_{3}$. Both $x_{13}$ and $x_{31}$ are neglected since they are associated with zeros in the $R H S$ and thus step 4 is done. However, the configuration selection satisfies to the pairing rules for the nominal system $G$ given in (24) and remaining algorithm steps of the proposed approach need to be performed to validate this configuration for the perturbed system. Thereafter, the information provided by the Push-Pull algorithm is used to determine the perturbation set $(\theta)$ on which the optimal configuration obtained from the nominal system $G$ is still valid. The perturbation set is defined ${ }^{40}$ as

$$
\theta=\left\{P_{i j} \mid C_{N}-C_{B} \cdot[A] \geq 0\right\}
$$

where $C_{B}$ is a row vector with elements equal to $\left|\phi_{i j}\right|+P_{i j}$ correspond to the BVS and $C_{N}$ is similar to $C_{B}$ except with the elements correspond to the non-BV while [A] denotes the matrix/vector obtained from the final tableau. Particularly, for this example

$$
\begin{gathered}
C_{B}=\left[\left|\phi_{12}\right|+P_{12},\left|\phi_{21}\right|+P_{21},\left|\phi_{33}\right|+P_{33},\right. \\
\left.\left|\phi_{13}\right|+P_{13},\left|\phi_{31}\right|+P_{31}\right] \\
C_{N}=\left[\left|\phi_{22}\right|+P_{22}\right]
\end{gathered}
$$

and

$$
A=\left[\begin{array}{lllll}
1 & 1 & 1 & -1 & -1
\end{array}\right]^{T}
$$

thus, the perturbation set $(\theta)$ is obtained as

$$
\begin{aligned}
\theta=\left\{P_{i j} \mid\right. & P_{13}-P_{12}-P_{21}+P_{22} \\
& \left.+P_{31}-P_{33}+0.5606 \geq 0\right\}
\end{aligned}
$$


Keep in mind that $P_{i j}$ in (27) are bounded sets according to (20) rather than certain values as of $c_{i j}^{\prime}$ in (8) in the marketing field. Furthermore, there are two zeros in the RHS of Table 1 therefore the degenerated phase has to be performed to modify the perturbation set $(\theta) .{ }^{40}$ Fortunately, the perturbation sets provided from the degenerated phase coincide with (27) and thus no modification is needed and step 5 of the proposed approach is completed.

Step 6 of the proposed approach is to calculate the bounds of the P's employing (21) and (22) except for those correspond to $\underline{\phi_{P i j}} \leq-1$. The bounds are to be used to determine the vertices to be utilized in the verification process. Thus, for

$$
G_{P}=G(0)+\delta G
$$

the bounds of the P's are found as

$$
\begin{aligned}
& -0.071 \leq P_{12} \leq 0.071 \\
& -0.115 \leq P_{13} \leq 0.115 \\
& -0.071 \leq P_{21} \leq 0.071 \\
& -0.115 \leq P_{22} \leq 0.115 \\
& -0.115 \leq P_{31} \leq 0.115
\end{aligned}
$$

and

$$
-0.054 \leq P_{33} \leq 0.054
$$


Following step 7 , the $\left(2^{(3 \times 3)-3}=64\right)$ vertices are formulated as

$$
v_{1}=\left[\begin{array}{l}
-0.071 \\
-0.115 \\
-0.071 \\
-0.115 \\
-0.115 \\
-0.054
\end{array}\right], v_{2}=\left[\begin{array}{c}
0.071 \\
-0.115 \\
-0.071 \\
-0.115 \\
-0.115 \\
-0.054
\end{array}\right], \cdots, v_{64}=\left[\begin{array}{l}
0.071 \\
0.115 \\
0.071 \\
0.115 \\
0.115 \\
0.054
\end{array}\right]
$$

Step 8 of the proposed approach is checking whether the vertices given in (29) satisfy the perturbation set condition given in (27). Since all the vertices satisfy the perturbation set, the optimality of the configuration selection obtained from the nominal model (24) in step 4 is still valid for the uncertain system defined by (28).

In order to validate the optimal pairing decision obtained from the above, two decentralized controllers, each consists of 3 IMC's controllers, are employed. The input and output variables of the perturbed system defined by $G(s)+\delta G$ are paired based on the optimal configuration and on the off-diagonal pairing (which is the remaining configuration after removing the ones which may lead to lose stability and integrity) as shown in Figure 2 and Figure 3, respectively.

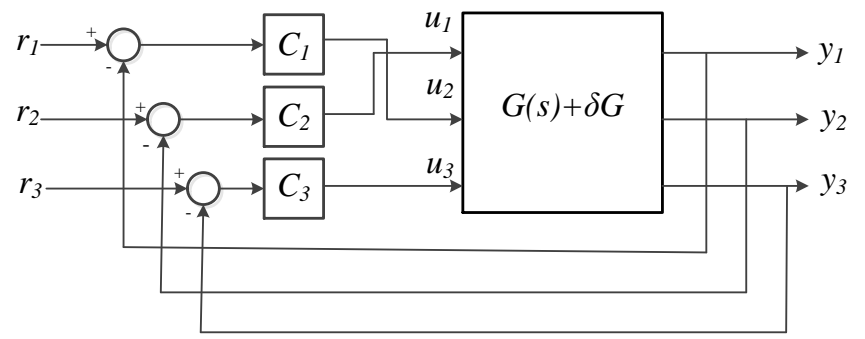

Figure 2: The closed-loop of the perturbed system and the decentralized controller based on the optimal configuration selection.

The $C^{\prime}$ 's and $K$ 's IMC controllers are selected based on the nominal system ${ }^{44}$ given in 


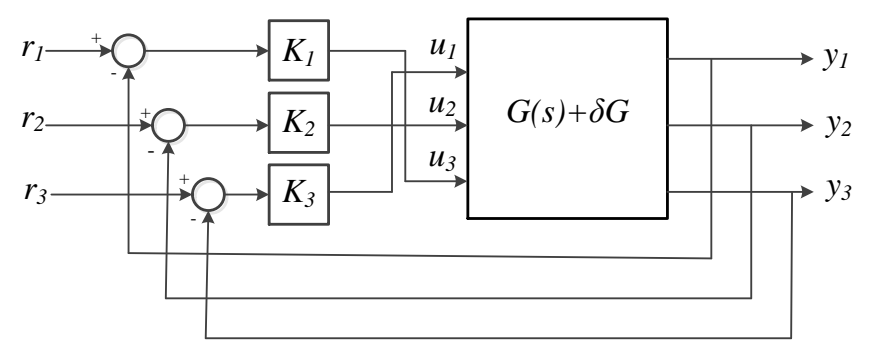

Figure 3: The closed-loop of the perturbed system and the decentralized controller based on the off-diagonal pairing.

(24) as

$$
C_{1}=\frac{g_{21}^{-1}}{2 s}, C_{2}=\frac{g_{12}^{-1}}{2 s} \text { and } C_{3}=\frac{g_{33}^{-1}}{2 s}
$$

where

$$
g_{21}=g_{12}=\frac{1.5}{(s+1)} \text { and } g_{33}=\frac{1.5}{(2 s+1)}
$$

while

$$
K_{1}=\frac{g_{31}^{-1}}{2 s}, K_{2}=\frac{g_{22}^{-1}}{2 s} \text { and } K_{3}=\frac{g_{13}^{-1}}{2 s}
$$

with

$$
g_{31}=g_{22}=g_{13}=\frac{1}{(s+1)}
$$

Figures 4 and 5 show the responses of the interaction-free, nominal and perturbed systems under the optimal and the off-diagonal pairing respectively, for the set-point changes in the references $r_{1}, r_{2}$ and $r_{3}$ at 10,60 and 110 sec. The perturbed systems are simulated by selecting 100 uniformly distributed random values of $\delta G$ that satisfy (25) and adding each to the nominal system gains of (24). By plotting the responses of the interaction-free system, the effect of the interaction on the responses under the used configurations could easily be observed. It is worth noting that the responses of the perturbed systems are closely around the response of nominal system under the optimal configuration as can be seen from the enlarged sample region in Figure 4. 

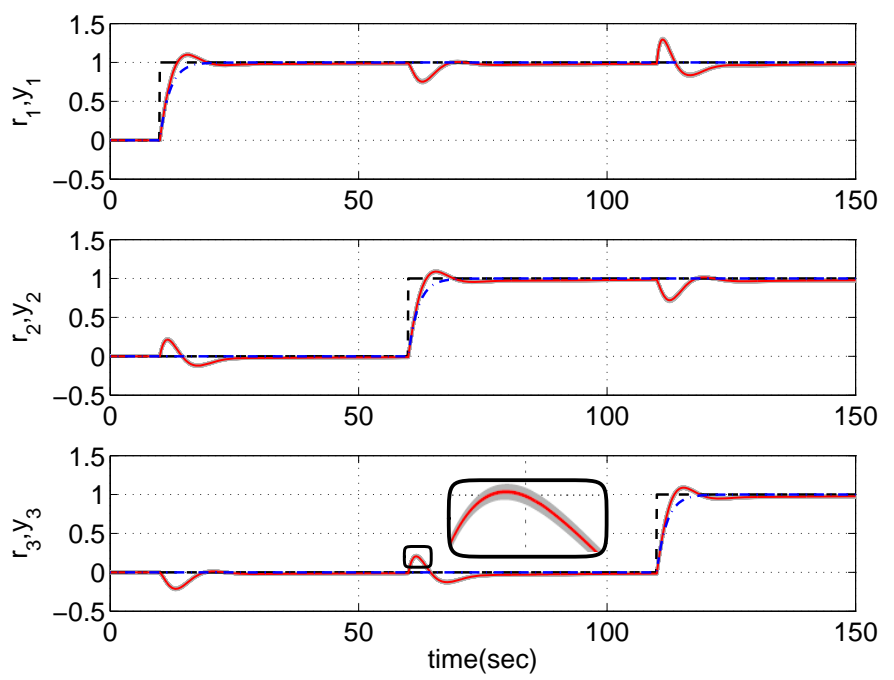

Figure 4: Set-point change responses of the interaction-free, nominal system and perturbed systems under optimal pairing. Dashed: the reference signals. Dash-dotted: response of the interaction-free system. Solid: response of the nominal system given in (24). Shade-line: responses of 100 perturbed systems.
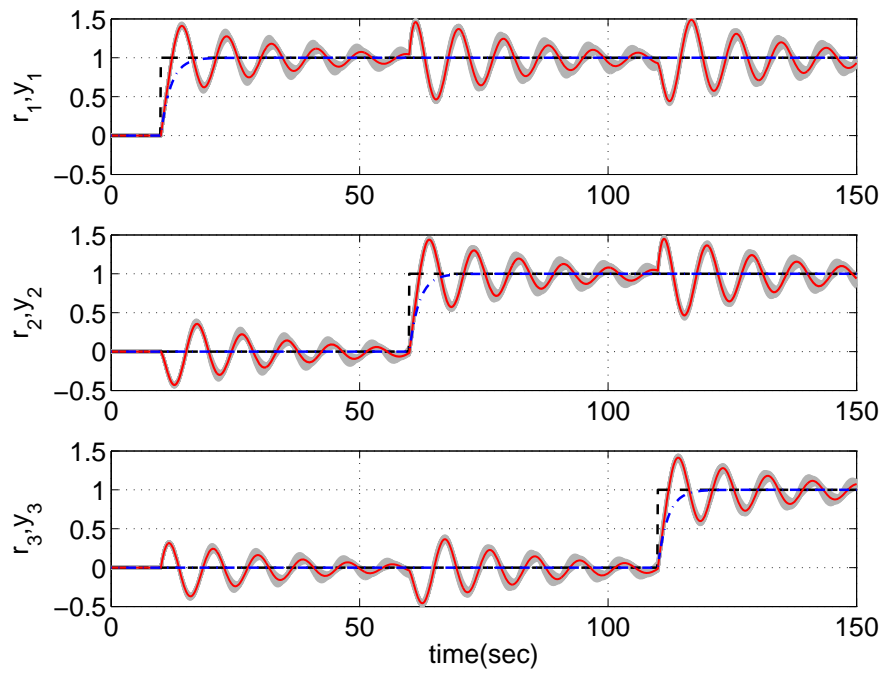

Figure 5: Set-point change responses of the interaction-free, nominal system and perturbed systems under off-diagonal pairing. Dashed: the reference signals. Dash-dotted: response of the interaction-free system. Solid: response of the nominal system given in (24). Shade-line: responses of 100 perturbed systems. 
The integral square error (ISE) of $e_{i}=y_{i}-r_{i}$ expressed by

$$
J=\sum_{i=1}^{n} \int_{0}^{t_{\text {end }}} e_{i}^{2} d t
$$

with $t_{\text {end }}=150 \mathrm{sec}$ and $n=3$ is used as a control performance indication. The $J$ values of the responses of the closed-loop systems for the different cases are depicted in the Table 2. Notice that, the interaction-free systems under both configurations have the same $J$ value which is quite expected from their responses in Figure 4 and Figure 5. Moreover, as $\delta G$ in (25) is bounded, the $J$ appears as an interval, $\left[\begin{array}{lll}J_{\min } & J_{\max }\end{array}\right]$, in the uncertain systems cases. The $J$ values of the nominal and uncertain systems under the optimal configuration

Table 2: ISE for the different closed-loop cases

\begin{tabular}{ccc}
\hline System & Configuration & $J$ \\
\hline interaction-free & optimal/off-diagonal & 3.152 \\
nominal & optimal & 4.201 \\
uncertain & optimal & {$[4.0484 .359]$} \\
nominal & off-diagonal & 16.316 \\
uncertain & off-diagonal & {$[14.05819 .877]$} \\
\hline
\end{tabular}

selection compared to the values under the off-diagonal pairing confirm the result of the proposed approach which states that the optimal configuration of the nominal system is still optimal for the systems perturbed by (25).

In order to give a glimpse on the case where the Push-Pull algorithm gives no solution, $\alpha=0.3$ in (25) with the same nominal system are processed. In this case, $\underline{\Phi_{P}}$ is obtained as

$$
\underline{\Phi_{P}}=\left(\begin{array}{ccc}
-6.584 & -2.312 & -3.120 \\
-2.312 & -3.120 & -5.597 \\
-3.120 & -5.597 & -1.806
\end{array}\right)
$$

As all the values are $<-1$ then all possible pairs violate the stability and integrity conditions 
and no pair can be suggested. Technically, there will be no enough data to formulate the initial tableau of the Push-Pull algorithm since all $\phi_{i j}$ correspond to the $\underline{\phi_{P i j}} \leq-1$ are to be removed at early stages of the algorithm. Consequently, the Push-Pull algorithm will give no answer. To motivate the instability concern that might occur, the simulation of the system under the optimal configuration selection in Figure 2 is re-performed for the new $\alpha$ and the results are presented in Figure 6. The results show satisfactory, sluggish as well as unstable perturbed systems responses. Obviously, the unstable responses draw the attention of the user for how they can be avoid. Suggesting no decentralized controller in the early stage of the proposed approach guides the user to look for other alternatives such as sparse or centralized controllers.
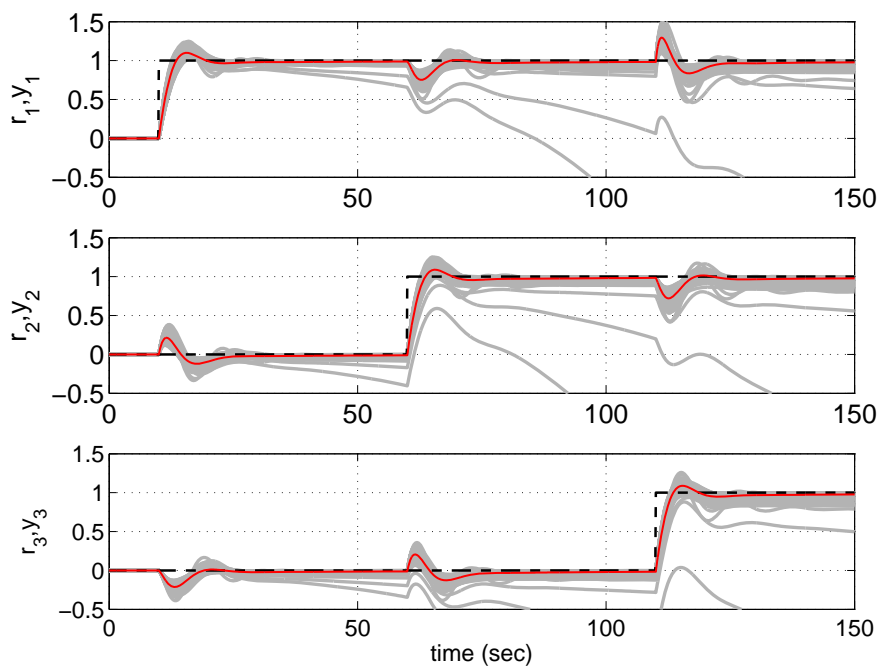

Figure 6: Set-point change responses of the nominal system and the new perturbed systems. Dashed: the reference signals. Solid: response of the nominal system given in (24). Shadeline: responses of new 100 perturbed systems. 


\subsection{Example 2}

Consider a steady-state gain matrix of ALSTOM gasifier plant ${ }^{45}$ given as

$$
G(0)=\left[\begin{array}{cccc}
0.0385 & -0.0427 & 0.0444 & -0.0474 \\
-0.1115 & -0.0297 & 0.0770 & -0.0142 \\
0.0327 & 0.8630 & 0.0477 & 0.5019 \\
0.0088 & 0.1284 & -0.1101 & -0.2834
\end{array}\right]
$$

and consider the plant to be subject to an additive uncertainty given by

$$
|\delta G| \leq \alpha|G(0)|
$$

with $\alpha=0.135$.

The $\Phi$ is obtained by employing step $\mathbf{1}$ as

$$
\Phi=\left(\begin{array}{cccc}
2.0244 & -19.5242 & 0.8513 & 4.4266 \\
0.5023 & -40.236 & 1.9544 & 45.8123 \\
98.952 & 0.1361 & 23.329 & 13.559 \\
-193.38 & 4.0186 & 11.459 & 0.378
\end{array}\right)
$$

Preforming step 2 gives the lower uncertainty bound values $\left(\underline{\Phi_{P}}\right)$ as in the following matrix

$$
\underline{\Phi_{P}}=\left(\begin{array}{cccc}
0.7412 & -47.5653 & -0.5290 & -1.3845 \\
-0.1557 & -81.0691 & -0.1485 & -0.8622 \\
-6.0296 & -0.3017 & 6.7447 & -8.8358 \\
-501.2177 & 0.0665 & -6.3258 & 0.1565
\end{array}\right)
$$


Consequently, any pair correspond to $\phi_{12}, \phi_{13}, \phi_{22}, \phi_{31}, \phi_{34}, \phi_{41}$ and $\phi_{43}$ are to be remove during the Push-Pull algorithm since their values are $<-1$. The optimal configuration selection and the perturbation set are obtained after performing the Push-Pull as in step 3. The final tableau is depicted in Table 3 . The optimal configuration selection is $\left(y_{1}-u_{3}\right)$,

Table 3: Final tableau

\begin{tabular}{cccc}
\hline BVS & $x_{23}$ & $x_{24}$ & $R H S$ \\
\hline$x_{13}$ & 1 & 1 & 1 \\
$x_{21}$ & 1 & 1 & 1 \\
$x_{32}$ & 0 & 1 & 1 \\
$x_{44}$ & 0 & 1 & 1 \\
$x_{42}$ & 0 & -1 & 0 \\
$x_{11}$ & -1 & -1 & 0 \\
$x_{33}$ & 0 & -1 & 0 \\
\hline
\end{tabular}

$\left(y_{2}-u_{1}\right),\left(y_{3}-u_{2}\right)$ and $\left(y_{4}-u_{4}\right)$ corresponds to the BVS in the final tableau excluding $x_{42}$, $x_{11}$ and $x_{33}$ that have value of zero in the RHS column and thus step 4 is completed. The perturbation set $(\theta)$ including the output of the degenerated phase is given by

$$
\begin{gathered}
\theta=\left\{P_{i j} \mid P_{11}-P_{13}-P_{21}+P_{23}+2.6351 \geq 0\right. \\
P_{11}-P_{13}-P_{21}+P_{24}-P_{32}+P_{33}+P_{42} \\
-P_{44}+73.327 \geq 0 \\
\left.P_{24}-P_{23}-P_{32}+P_{33}+P_{42}-P_{44}+70.691 \geq 0\right\}
\end{gathered}
$$

Obtaining the perturbation set $(\theta)$ ends step 5 of the proposed approach. In step $\mathbf{6}$, the bounds in (21) and (22) are calculated to formulate the vertices of the polytopic model as in step 7. For the uncertain system

$$
G_{P}=G(0)+\delta G
$$


the uncertainty bounds of the P's (except for those associated to $\underline{\phi_{P i j}} \leq-1$ ) are found as

$$
\begin{aligned}
& -1.293 \leq P_{11} \leq 1.293 \\
& -4.426 \leq P_{14} \leq 5.8111 \\
& -0.502 \leq P_{21} \leq 0.657 \\
& -1.954 \leq P_{23} \leq 2.102 \\
& -45.812 \leq P_{24} \leq 46.674 \\
& -0.136 \leq P_{32} \leq 0.437 \\
& -16.584 \leq P_{33} \leq 16.584 \\
& -3.952 \leq P_{42} \leq 3.952
\end{aligned}
$$

and

$$
-0.221 \leq P_{44} \leq 0.221
$$

The verification process, step 8 , reveals that there are many among the $\left(2^{(4 \times 4)-7}=512\right)$ 
vertices do not satisfy the perturbation set $(\theta)$. For example the vertices

$$
v_{159}=\left[\begin{array}{c}
-1.293 \\
5.811 \\
-0.502 \\
-1.954 \\
46.674 \\
0.437 \\
16.584 \\
3.952 \\
-0.221
\end{array}\right], v_{470}=\left[\begin{array}{c}
1.293 \\
5.811 \\
0.657 \\
-1.954 \\
46.674 \\
-0.136 \\
16.584 \\
-3.952 \\
0.221
\end{array}\right] \text { and } v_{478}=\left[\begin{array}{c}
1.293 \\
5.811 \\
0.657 \\
-1.954 \\
46.674 \\
0.437 \\
16.584 \\
-3.952 \\
0.221
\end{array}\right]
$$

do not satisfy the inequality

$$
P_{11}-P_{13}-P_{21}+P_{23}+2.6351 \geq 0
$$

Therefore, the optimality of the configuration selection obtained from the nominal system $G$ given by (30) is not guaranteed with respect to the perturbed system $G_{P}$ given by (33). To conclude, the configuration obtained based on the nominal system satisfies the closed-loop stability and integrity requirements but not minimize the loop interaction of the perturbed system.

For more thorough discussion, a particular uncertain system which belongs to the uncertainty set defined by (33) is considered as

$$
G_{P 1}=G(0)+\delta G_{1}
$$


where

$$
\delta G_{1}=0.135|G(0)| \times\left[\begin{array}{cccc}
1 & 1 & -1 & 1 \\
1 & 1 & 1 & -1 \\
1 & -1 & -1 & 1 \\
-1 & 1 & 1 & -1
\end{array}\right]
$$

with $\times$ denoting element-by-element multiplication.

The RIA matrix of the uncertain system $\left(G_{P 1}\right)$, which is given by

$$
\Phi_{P 1}=\left(\begin{array}{cccc}
1.1187 & -29.8732 & 1.2286 & 7.7772 \\
0.9413 & -40.8102 & 1.0474 & 45.369 \\
62.9303 & 0.2411 & 28.2304 & 5.9247 \\
-363.0956 & 2.9367 & 33.9005 & 0.3887
\end{array}\right)
$$

shows that the pairs $\left(y_{1}-u_{3}\right)$ and $\left(y_{2}-u_{1}\right)$ obtained based on the nominal system (30) should be replaced by $\left(y_{1}-u_{1}\right)$ and $\left(y_{2}-u_{3}\right)$, respectively. In other words, using $\phi_{11}$ and $\phi_{23}$ in $\Phi_{P 1}$ results in a less value for the summation in pairing rule $(\mathrm{d})$ than $\phi_{13}$ and $\phi_{21}$. Therefore, the optimal configuration selection for the uncertain system $\left(G_{P 1}\right)$ is $\left(y_{1}-u_{1}\right),\left(y_{2}-u_{3}\right)$, $\left(y_{3}-u_{2}\right)$ and $\left(y_{4}-u_{4}\right)$ rather than the previous configuration obtained based on the nominal system. In order to confirm and compare this result with a different approach, the optimal pairing of nominal system $(G(0))$ and $G_{P 1}$ are analyzed following the BAB method ${ }^{19}$. The application of the method could confirm the new optimal pairing for $G_{P 1}$. Nevertheless, the conditions of the closed-loop stability and integrity of the uncertain system $\left(G_{P 1}\right)$ under previous configuration are fulfilled since $\phi_{P 13}, \phi_{P 21}, \phi_{P 32}$ and $\phi_{P 44}$ are not close to or $\leq-1$.

In short, the previous configuration obtained from the nominal model $G$ satisfies closedloop stability and integrity requirements of the uncertain system $\left(G_{P 1}\right)$ but does not minimize the loop interaction effect. This result coincides with the prediction of the proposed approach based on lower uncertainty bounds analysis and vertices verification. 


\section{Conclusion and Future Work}

An approach for automated control configuration selection considering the system uncertainties is proposed. The optimal configuration based on the nominal system is obtained systematically by formulating the overall interaction rule of the RIA as an assignment problem and solving it using the Push-Pull algorithm. That has been done after removing any pair that does not satisfy the stability and integrity conditions utilizing the uncertainty bound of the RIA elements. The proposed approach employs both the perturbation set and the uncertainty bounds of the absolute RIA elements to verify whether the obtained optimal configuration is still optimum under presence of a specific perturbation or a change is expected. The approach has been validated by simulation examples for a $3 \times 3$ system and the $4 \times 4$ ALSTOM gasifier plant, respectively.

The presented approach offers a novel tool to suggest a control configuration for a system subject to perturbation, yet there are some shortcomings that should be addressed. Despite the fact that the Push-Pull algorithm solves the optimization problem only once, the number of vertices increases relatively with the system size leading to a prolonged time in the validation process for large systems. Besides, the uncertainty bounds of the RIA elements need to be tightened to overcome the possible conservatism in the pairing decision in real world applications. However, the proposed algorithm is independent on those bounds in the sense that whenever new bounds are found, they can be directly integrated.

The proposed approach assures the pairing optimality preservation if all model vertices satisfy the perturbation set. A systematic procedure to be developed investigating the effect of the vertices out of perturbation set to assure the optimality change occurrence. Moreover, knowing the amount of the uncertain gains $\delta G$ which causes a change in the optimal pairing and knowing the new optimal pairing for the uncertain system are also of importance. Therefore, more elaboration on the proposed approach in that direction is planned as future work. 


\section{Appendix}

Deriving the expression of $\frac{\partial \phi_{i j}}{\partial g_{k l}}$

The variation of $\phi_{i j}\left(\partial \phi_{i j}\right)$ with respect to only $g_{i j}\left(\partial g_{i j}\right)$ is given by Zhu. ${ }^{17}$ Thus, generalization of the $\phi_{i j}$ variation with respect to variation of all other $g^{\prime} s$ is essential for calculating the uncertainty bounds in (14). Employing (5), a relation between $\phi_{i j}$ and $g_{k l}$ can be found to derive the term $\frac{\partial \phi_{i j}}{\partial g_{k l}}$. Nevertheless, going in that direction is obviously tedious. Instead, the

chain rule and the expressions $\frac{\partial \lambda_{i j}}{\partial g_{k l}}$ by Chen and Seborg ${ }^{9}$ are used to derive the expressions of $\frac{\partial \phi_{i j}}{\partial g_{k l}}$ where $\lambda_{i j}$ denotes the $(i j)$-element in the RGA matrix.

The derivation steps are as follows; utilizing the chain rule, $\frac{\partial \phi_{i j}}{\partial g_{k l}}$ can be written as

$$
\frac{\partial \phi_{i j}}{\partial g_{k l}}=\frac{\partial \phi_{i j}}{\partial \lambda_{i j}} \cdot \frac{\partial \lambda_{i j}}{\partial g_{k l}}
$$

From (5), a relation between $\phi_{i j}$ and $\lambda_{i j}$ is expressed ${ }^{17}$ as

$$
\phi_{i j}=\frac{1}{\lambda_{i j}}-1
$$

then

$$
\frac{\partial \phi_{i j}}{\partial \lambda_{i j}}=-\frac{1}{\lambda_{i j}^{2}}
$$

Since $\frac{\partial \lambda_{i j}}{\partial g_{k l}}$ is given ${ }^{9}$ as in (38),

$$
\frac{\partial \lambda_{i j}}{\partial g_{k l}}= \begin{cases}\frac{\lambda_{i j}\left(1-\lambda_{i j}\right)}{g_{i j}} & i=k \text { and } j=l \\ -\frac{\lambda_{i j} \lambda_{k l}}{g_{k l}} & i=k \text { or } j=l \\ \frac{(-1)^{i+j+k+l} g_{i j} \operatorname{det}\left(G^{i j, k l}\right)}{\operatorname{det}(G)}-\frac{\lambda_{i j} \lambda_{k l}}{g_{k l}} & i \neq k \text { and } j \neq l\end{cases}
$$

where $G^{i j, k l}$ means the submatrix of $G$ with rows $i$ and $k$ and columns $j$ and $l$ are removed, 
the $\frac{\partial \phi_{i j}}{\partial g_{k l}}$ can be written as in (39).

$$
\frac{\partial \phi_{i j}}{\partial g_{k l}}=\frac{\partial \phi_{i j}}{\partial \lambda_{i j}} \cdot \frac{\partial \lambda_{i j}}{\partial g_{k l}}= \begin{cases}-\frac{\left(1-\lambda_{i j}\right)}{\lambda_{i j} g_{i j}} & i=k \text { and } j=l \\ \frac{\lambda_{k l}}{\lambda_{i j} g_{k l}} & i=k \text { or } j=l \\ -\frac{(-1)^{i+j+k+l} g_{i j} \operatorname{det}\left(G^{i j, k l}\right)}{\lambda_{i j}^{2} \operatorname{det}(G)}+\frac{\lambda_{k l}}{\lambda_{i j} g_{k l}} & i \neq k \text { and } j \neq l\end{cases}
$$

Finally, a substitution of

$$
\lambda_{i j}=\frac{1}{\phi_{i j}+1}
$$

(rearranged from (36)) in (39) is performed to obtain the final expressions of $\frac{\partial \phi_{i j}}{\partial g_{k l}}$ as in (11)

\section{Alternative uncertainty bounds of $\phi_{P i j}$}

Following the approach introduced by Chen and Seborg, ${ }^{9}$ the uncertainty bounds of the perturbed RGA elements $\left(\lambda_{P i j}\right)$ can be written as

$$
\overbrace{\lambda_{i j}-\sum_{k=1}^{n} \sum_{l=1}^{n} \mid\left(\frac{\partial \lambda_{i j}}{\partial g_{k l}}\right)_{G}\left(\delta g_{k l}\right)}^{\lambda_{P i j}} \mid \leq \lambda_{P i j} \leq \underbrace{\underbrace{n}_{l=1}}_{\frac{\lambda_{P i j}}{\lambda_{i j}+\sum_{k=1}^{n} \sum_{l=1}^{n}\left|\left(\frac{\partial \lambda_{i j}}{\partial g_{k l}}\right)_{G}\left(\delta g_{k l}\right)\right|}}
$$

where $\lambda_{i j}$ is the $i j$-element in RGA calculated based on the nominal system $G$ and $\frac{\partial \lambda_{i j}}{\partial g_{k l}}$ is defined in (38).

Employing (5), the uncertainty bounds of $\phi_{P i j}$ can be calculated as

$$
\overbrace{\frac{1}{\lambda_{P i j}}-1}^{\phi_{P i j}} \leq \phi_{P i j} \leq \underbrace{\frac{1}{\lambda_{P i j}}}_{\frac{1}{\bar{\phi}_{P i j}}-1}
$$

However, the user has to be careful if the uncertainty bounds of the $\lambda_{P i j}$ crosses the zero, 
in such a case the $\phi_{P i j}$ uncertainty bounds will be the union of two sets as

$$
\left[-\infty, \overline{\phi_{P i j}}\right] \cup\left[\underline{\phi_{P i j}}, \infty\right]
$$

and the user has to select the one which encloses the nominal value $\left(\phi_{i j}\right)$. For example, consider a steady-state gain matrix of a binary distillation column ${ }^{46}$ given as

$$
G(0)=\left[\begin{array}{ccc}
0.66 & -0.61 & -0.0049 \\
1.11 & -2.36 & -0.012 \\
-33.68 & 46.2 & 0.87
\end{array}\right]
$$

The RGA and the RIA are calculated as

$$
\Lambda=\left[\begin{array}{ccc}
1.9454 & -0.6737 & -0.2718 \\
-0.6643 & 1.8991 & -0.2348 \\
-0.2811 & -0.2254 & 1.5065
\end{array}\right], \Phi=\left[\begin{array}{ccc}
-0.4860 & -2.4844 & -4.6794 \\
-2.5053 & -0.4734 & -5.2598 \\
-4.5573 & -5.4361 & -0.3362
\end{array}\right]
$$

The uncertainty bounds of the RGA caused by an additive uncertainty $\delta G \leq 0.1|G(0)|$ are given using (40) as

$$
\Lambda_{P}=\left[\begin{array}{ccc}
1.2097 \leq \lambda_{11} \leq 2.6812 & -1.2644 \leq \lambda_{12} \leq-0.0829 & -0.7450 \leq \lambda_{13} \leq 0.2015 \\
-1.2534 \leq \lambda_{21} \leq-0.0753 & 1.0061 \leq \lambda_{22} \leq 2.7921 & -0.5464 \leq \lambda_{23} \leq 0.0769 \\
-0.7606 \leq \lambda_{31} \leq 0.1984 & -0.5361 \leq \lambda_{32} \leq 0.0853 & 1.2013 \leq \lambda_{33} \leq 1.8118
\end{array}\right]
$$

By applying (41) and (42), the uncertainty bounds of $\Phi_{P}$ are obtained as

$$
\Phi_{P}=\left[\begin{array}{ccc}
-0.6270 \leq \phi_{11} \leq-0.1734 & -13.0668 \leq \phi_{12} \leq-1.7909 & -\infty \leq \phi_{13} \leq-2.3422 \\
-14.2791 \leq \phi_{21} \leq-1.7979 & -0.6418 \leq \phi_{22} \leq-0.0061 & -\infty \leq \phi_{23} \leq-2.8302 \\
-\infty \leq \phi_{31} \leq-2.3147 & -\infty \leq \phi_{32} \leq-2.8653 & -0.4481 \leq \phi_{33} \leq-0.1676
\end{array}\right]
$$


Furthermore, to make an accuracy comparison, in term of the absolute error $(A . E)$, between the $\Phi_{p}$ uncertainty bounds derived in Section 5 (denoted by $\Phi_{S 5}$ ) and the ones given here $\left(\Phi_{A 2}\right)$, system (43) with an additive perturbation $\delta G=0.1 \times|G(0)|$ is employed. The absolute error committed by $\Phi_{S 5}$ is

$$
A . E_{S 5}=\left|\Phi(G(0)+\delta G)-\Phi_{S 5}(G(0)+\delta G)\right|=\left[\begin{array}{lll}
0.0361 & 0.1802 & 55.1988 \\
0.1468 & 0.0101 & 1.4776 \\
2.2692 & 0.3973 & 0.0281
\end{array}\right]
$$

while the absolute error committed by $\Phi_{A 2}$ is

$$
A . E_{A 2}=\left|\Phi(G(0)+\delta G)-\Phi_{A 2}(G(0)+\delta G)\right|=\left[\begin{array}{lll}
0.0406 & 0.2062 & 55.6625 \\
0.1748 & 0.0134 & 0.9354 \\
2.7163 & 0.3746 & 0.0263
\end{array}\right]
$$

A binary comparison of the both result

$$
A \cdot E_{S 5}<A . E_{A 2}=\left[\begin{array}{ccc}
1 & 1 & 1 \\
1 & 1 & 0 \\
1 & 0 & 0
\end{array}\right]
$$

shows that the uncertainty bounds derived in Section 5 gives lower absolute error than the one given here in 6 elements out of 9 . However, a general conclusion about which approach is more accurate can not be drown for just one example.

\section{Acknowledgments}

This work has been partially funded by: a) the Horizon 2020 OPTi project under the Grant Agreement No. 649796, b) the Horizon 2020 DISIRE project under the Grant Agreement No. 636834, c) the WARP project from the PiiA postdoc program. 


\section{References}

(1) Seborg, D. E.; Mellichamp, D. A.; Edgar, T. F.; Doyle III, F. J. Process dynamics and control; John Wiley \& Sons, 2010.

(2) Luppi, P. A.; Zumoffen, D. A. R.; Basualdo, M. S. Decentralized plantwide control strategy for large-scale processes. Case study: Pulp mill benchmark problem. Computers $\&$ Chemical Engineering 2013, 52, 272-285.

(3) Zumoffen, D.; Basualdo, M. Improvements in fault tolerance characteristics for large chemical plants: 1 . Waste water treatment plant with decentralized control. Industrial $\&$ Engineering Chemistry Research 2008, 47, 5464-5481.

(4) Skogestad, S.; Postlethwaite, I. Multivariable feedback control: analysis and design; Wiley New York, 2007; Vol. 2.

(5) Christofides, P. D.; Scattolini, R.; de la Pena, D. M.; Liu, J. Distributed model predictive control: A tutorial review and future research directions. Computers $\&$ Chemical Engineering 2013, 51, 21-41.

(6) Rijnsdorp, J. Interaction in two-variable control systems for distillation columns-I: Theory. Automatica 1965, 3, 15-28.

(7) Bristol, E. On a new measure of interaction for multivariable process control. Automatic Control, IEEE Transactions on 1966, 11, 133-134.

(8) Khaki-Sedigh, A.; Moaveni, B. Control configuration selection for multivariable plants; Springer, 2009; Vol. 391.

(9) Chen, D.; Seborg, D. E. Relative gain array analysis for uncertain process models. AIChE journal 2002, 48, 302-310.

(10) Kariwala, V.; Skogestad, S.; Forbes, J. F. Relative gain array for norm-bounded uncertain systems. Industrial $\&$ engineering chemistry research 2006, 45, 1751-1757. 
(11) Moaveni, B.; Sedigh, A. K. Further Theoretical Results on "Relative Gain Array for Norm-Bounded Uncertain Systems". Industrial \& Engineering Chemistry Research 2007, 46, 8288-8289.

(12) Kadhim, A. M.; Birk, W.; Gustafsson, T. Relative gain array variation for norm bounded uncertain systems. Decision and Control (CDC), 2015 IEEE 54th Annual Conference on. 2015; pp 5959-5965.

(13) Castaño, M.; Birk, W. A new approach to the dynamic RGA analysis of uncertain systems. IEEE International Conference on Computer-Aided Control Systems, 2008. CACSD 2008. 2008; pp 365-370.

(14) Morari, M. Interactions between process design and process control; Pergamon Oxford, 1992; pp 3-16.

(15) Niederlinski, A. A heuristic approach to the design of linear multivariable interacting control systems. Automatica 1971, 7, 691-701.

(16) Zhu, Z.-X.; Jutan, A. Loop decomposition and dynamic interaction analysis of decentralized control systems. Chemical engineering science 1996, 51, 3325-3335.

(17) Zhu, Z.-X. Variable pairing selection based on individual and overall interaction measures. Industrial \& engineering chemistry research 1996, 35, 4091-4099.

(18) Kariwala, V.; Cao, Y. Efficient branch and bound methods for pairing selection. IFAC Proceedings Volumes 2008, 41, 12935-12940.

(19) Kariwala, V.; Cao, Y. Branch and bound method for multiobjective pairing selection. Automatica 2010, 46, 932-936.

(20) Kookos, I.; Lygeros, A. An algorithmic method for control structure selection based on the RGA and RIA interaction measures. Chemical Engineering Research and Design 1998, 76, 458-464. 
(21) Fatehi, A. Automatic pairing of large scale MIMO plants using normalised RGA. International Journal of Modelling, Identification and Control 2011, 14, 37-45.

(22) Nobakhti, A. Improved profitability of a pulping mill through progressive controller structure expansion. Proceedings of the Institution of Mechanical Engineers, Part I: Journal of Systems and Control Engineering 2010, 224, 771-787.

(23) Skogestad, S. Control Structure Design for Complete Chemical Plants. Computers $\&$ Chemical Engineering 2004, 28, 219-234.

(24) Birk, W. Towards Incremental Control Structure Improvement. Proc of 46th IEEE Conference on Decision and Control, New Orleans, USA. 2007.

(25) Salgado, M.; Conley, A. MIMO interaction measure and controller structure selection. International Journal of Control 2004, 77, 367-383.

(26) Wittenmark, B.; Salgado, M. E. Hankel-Norm Based Interaction Measure for InputOutput Pairing. Proc. of the IFAC World Congress. 2002.

(27) Birk, W.; Medvedev, A. A Note on Gramian-Based Interaction Measures. Proc. of the European Control Conference (ECC). 2003.

(28) Shaker, H. R.; Tahavori, M. Control configuration selection for bilinear systems via generalised hankel interaction index array. International Journal of Control 2015, 88, $30-37$.

(29) Castaño, M.; Birk, W.; Asplund, P. Control configuration selection for integrating processes using graphs. 2015 IEEE Conference on Control Applications (CCA). 2015; pp 1606-1611.

(30) Shaker, H. R.; Tahavori, M. Frequency-interval control configuration selection for multivariable bilinear systems. Journal of Process Control 2013, 23, 894 - 904. 
(31) Gawronski, W.; Juang, J.-N. Model reduction in limited time and frequency intervals. International Journal of Systems Science 1990, 21, 349-376.

(32) Halvarsson, B.; Castaño, M.; Birk, W. Uncertainty bounds for Gramian-based interaction measures. 14th WSEAS International Conference on Systems 2010,

(33) Castaño, M.; Birk, W. Bounds on a gramian-based interaction measure for robust control structure selection. IEEE International Conference on Control \& Automation 2011,

(34) Castaño, M.; Birk, W.; Halvarsson, B. Empirical approach to robust gramian-based analysis of process interactions in control structure selection. Decision and Control and European Control Conference (CDC-ECC), 2011 50th IEEE Conference on. 2011.

(35) Castaño, M.; Birk, W. Estimation of gramian-based interaction measures for weakly nonlinear systems. Control Conference (ECC), 2015 European. 2015; pp 2438-2443.

(36) Castaño, M.; Birk, W. On the selection of control configurations for uncertain systems using gramian-based Interaction Measures.

(37) Castaño, M.; Birk, W. Model based and empirical approaches to robust control structure selection based on the H2-norm. Reglermöte. 2012.

(38) Birk, W.; Castaño, M.; Johansson, A. An application software for visualization and control configuration selection of interconnected processes. Control Engineering Practice 2014, 26, 188-200.

(39) Kasana, H. S.; Kumar, K. D. Introductory operations research: theory and applications; Springer Science \& Business Media, 2013.

(40) Adlakha, V.; Arsham, H. Managing cost uncertainties in transportation and assignment problems. Advances in Decision Sciences 1998, 2, 65-104. 
(41) Arsham, H.; Kahn, A. A refined simplex algorithm for the classical transportation problem with application to parametric analysis. Mathematical and Computer Modelling 1989, 12, 1035-1044.

(42) Arsham, H.; Cimperman, G.; Damij, N.; Damij, T.; Grad, J. A computer implementation of the Push-and-Pull algorithm and its computational comparison with LP simplex method. Applied mathematics and computation 2005, 170, 36-63.

(43) Toscano, R. Structured Controllers for Uncertain Systems: A Stochastic Optimization Approach; Springer Science \& Business Media, 2013.

(44) Bequette, B. W. Process control: modeling, design, and simulation; Prentice Hall Professional, 2003.

(45) Kariwala, V.; Forbes, J. F.; Meadows, E. S. Block relative gain: Properties and pairing rules. Industrial $\&$ engineering chemistry research 2003, 42, 4564-4574.

(46) Ogunnaike, B.; Lemaire, J.; Morari, M.; Ray, W. Advanced multivariable control of a pilot-plant distillation column. AIChE Journal 1983, 29, 632-640. 


\section{Graphical TOC Entry}

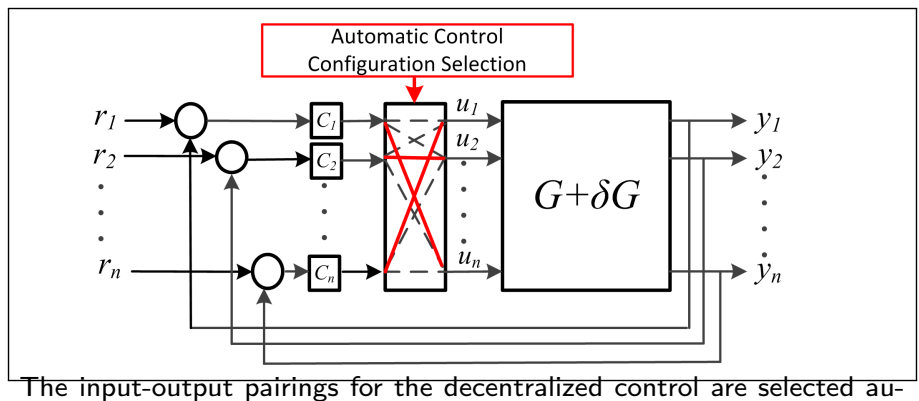

tomatically taking the system uncertainties into consideration. 